DOI: $10.1002 /$ ((please add manuscript number))

Article type: Full Paper

\title{
Semiconductor-based Photoelectrochemical Water Splitting at the Limit of Very Wide Depletion Region
}

Mingzhao Liu*, John L. Lyons, Danhua Yan, and Mark S. Hybertsen

Dr. M. Z. Liu, Dr. J. L. Lyons, D. H. Yan, Dr. M. S. Hybertsen

Center for Functional Nanomaterials, Brookhaven National Laboratory, Upton, NY 11973, United States

E-mail: mzliu@bnl.gov

Keywords: photoelectrochemical water splitting; strontium titanate; indirect band gap semiconductor; graded doping

In semiconductor-based photoelectrochemical (PEC) water splitting, carrier separation and delivery largely relies on the depletion region formed at the semiconductor/water interface. As a Schottky junction device, the trade-off between photon collection and minority carrier delivery remains a persistent obstacle for maximizing the performance of a water splitting photoelectrode. Here, it is demonstrated that the PEC water splitting efficiency for an $n$ $\mathrm{SrTiO}_{3}(n-\mathrm{STO})$ photoanode is improved very significantly despite its weak indirect band gap optical absorption $\left(\alpha<10^{4} \mathrm{~cm}^{-1}\right)$, by widening the depletion region through engineering its doping density and profile. Graded doped $n-\mathrm{SrTiO}_{3}$ photoanodes are fabricated with their bulk heavily doped with oxygen vacancies but their surface lightly doped over a tunable depth of a few hundred nanometers, through a simple low temperature re-oxidation technique. The graded doping profile widens the depletion region to over $500 \mathrm{~nm}$, thus leading to very efficient charge carrier separation and high quantum efficiency ( $>70 \%)$ for the weak indirect transition. This simultaneous optimization of the light absorption, minority carrier (hole) delivery, and majority carrier (electron) transport by means of a graded doping architecture may be useful for other indirect band gap photocatalysts that suffer from a similar problem of weak optical absorption. 


\section{Introduction}

Illuminating a direct pathway from solar energy to clean chemical fuel, photocatalytic water splitting has attracted significant attention over the past five decades. ${ }^{[1,2]}$ For a water splitting device that is based on a semiconductor photoelectrode, it is the space charge region (SCR) formed at the semiconductor side of the aqueous interface that dictates the separation, transport, and recombination dynamics of the photogenerated carriers. ${ }^{[3]}$ In most cases, majority carriers are depleted from the SCR, thus forming a depletion region; so that the device performance is essentially determined by the relaxation and transport of minority carriers. ${ }^{[4]}$ For $n$-type metal oxides that are typically used for the oxygen evolving photoanode, their low majority carrier (electron) mobility demands a high doping level $\left(10^{18}\right.$ $-10^{19} \mathrm{~cm}^{-3}$ ) to achieve low ohmic loss levels. Correspondingly, this suppresses the depletion width to a few tens of nanometers. The thin depletion layer makes it difficult to effectively utilize incident light, which is typically absorbed over a much longer distance. ${ }^{[5-7]}$ Apart from the popular solution of nanostructuring the photocatalyst, ${ }^{[8,9]}$ graded doping in bulk material has been proposed to broaden the depletion region without introducing additional surface defects. ${ }^{[10,11]}$ For direct band gap semiconductors such as $\mathrm{BiVO}_{4}$, the creation of an $n^{+}-n$ homojunction helps to match the depletion width with the photon attenuation depth $d_{p}$ (the depth that achieves optical density 1 for incident photon) of $\sim 100 \mathrm{~nm}$, and improves the external quantum efficiency up to $60 \%{ }^{[10]}$ To date, it remains an open question whether this strategy can be extended to metal oxide photocatalysts with indirect band gaps, such as $\alpha$ $\mathrm{Fe}_{2} \mathrm{O}_{3}, \mathrm{WO}_{3}$, and many perovskites, which have much weaker optical absorption. ${ }^{[12-18]}$

As a model perovskite material, strontium titanate $\left(\mathrm{SrTiO}_{3}, \mathrm{STO}\right)$ is among the earliest studied indirect band gap photocatalysts. ${ }^{[19,20]}$ With its indirect band gap at $3.25 \mathrm{eV}(380 \mathrm{~nm})$ and direct band gap at $3.75 \mathrm{eV}(330 \mathrm{~nm})$ (Figure 1a and its inset), STO is one of few photocatalysts that are capable of overall water splitting without external electrical bias due to its very low flat band potential. ${ }^{[19-21]}$ The density functional theory (DFT) band structure of 
STO is presented in the inset of Figure 1a, showing the indirect and direct transitions. The material can be readily $n$-type doped for a water oxidation photoanode, either by heating in a reducing environment or by doping with niobium. ${ }^{[22,23]}$ It was demonstrated by Wrighton, et al., that the quantum yield for water splitting over a heavily $n$-doped STO photoanode was only $\sim 10 \%$ for excitation at $3.5 \mathrm{eV}(354 \mathrm{~nm})$, a photon energy that remains well above the indirect band edge. ${ }^{[19]}$ The problem is clearly due to the weak optical absorption of the indirect transition (Figure 1a). ${ }^{[17]}$ In this letter we perform a systematic study on photoelectrochemical water splitting over single crystalline $n$-type STO photoanodes with different doping concentrations and profiles. The quantum efficiency arising from indirect transitions is improved substantially by lowering the doping concentration. Most significantly, we find that the depletion region is widened beyond $500 \mathrm{~nm}$ by creating a region of relatively low doping over the bulk $n$-STO. This is shown to improve the incident photon to current efficiency (IPCE) to over $60 \%$ for excitation at $3.5 \mathrm{eV}(354 \mathrm{~nm})$ and to over $70 \%$ for excitation at $3.6 \mathrm{eV}(344 \mathrm{~nm})$.

\section{Results and Discussion}

\subsection{PEC water splitting over uniformly doped $n$-STO photoanodes}

The $n$-STO photoanodes, either doped by niobium or oxygen vacancies, exhibit near ideal polarizable behavior in dark, i.e., passing negligible dark current within the $0-1.5 \mathrm{~V}_{\mathrm{RHE}}$ potential window (Figure 1b). Under the simulated AM1.5 solar radiation, all $n$-STO photoanodes deliver anodic photocurrent, with the onset potential at around $0 \mathrm{~V}_{\text {RHE}}$. The photocurrent saturates quickly as the electrode potential becomes more anodic, demonstrating an efficient carrier separation process and reflecting STO's negligible exciton binding energy that is due to its very large dielectric constant ( $\epsilon=300$ for STO). The donor density $N_{D}$, or more precisely, the density of charge that can be removed from donors under depletion conditions in each photoanode is determined by the Mott-Schottky relation, 


$$
\frac{1}{C_{S c}^{2}}=\frac{2}{e \epsilon \epsilon_{0} N_{D}}\left(E-E_{F B}-k_{B} T / e\right)
$$

where $e$ is the magnitude of the elementary charge, $\epsilon$ the dielectric constant, $\epsilon_{0}$ the vacuum permittivity, and $k_{B} T$ the thermal energy (Figure 1b). Due to the relatively low doping level, the dielectric constant $\epsilon$ is assumed to be equal to the one of pristine STO. The assumption is verified as it produces a donor density that is consistent with electrical transport measurements (Supporting Information). The space-charge capacitance per unit area $C_{s c}$ is measured by electrochemical impedance spectroscopy (Supporting Information, Figure S2). Consistent with the observed photocurrent onset potential, the flat band potential $E_{F B}$ is found to be about $-0.03 \mathrm{~V}_{\mathrm{RHE}}$, with little dependence on the doping concentration.

The water splitting IPCE, defined as the ratio of the number of collected photogenerated electrons to the number of incident photons, is measured for $n$-STO photoanodes with different dopants (either niobium or oxygen vacancies) and doping densities, versus the electrode potential in the spectral range of $300-400 \mathrm{~nm}$ (Figure 2). For all samples the IPCE is high for direct band edge excitation $(\lambda<330 \mathrm{~nm})$ but drops quickly as the excitation energy decreases, due to the weak optical absorption of the indirect transitions. The IPCE plots show strong dependence on the doping concentration, with higher efficiencies achieved for $n$-STO with lower donor density across the entire spectral range. For instance, an STO:Nb photoanode with $N_{D}=3.3 \times 10^{19} \mathrm{~cm}^{-3}$ achieves $66 \%$ IPCE at $310 \mathrm{~nm}$ but merely $3.8 \%$ at $360 \mathrm{~nm}$ (Figure 2a, $\left.E=1.23 \mathrm{~V}_{\mathrm{RHE}}\right)$, which increase to $79 \%(310 \mathrm{~nm})$ and $8.3 \%(360 \mathrm{~nm})$ for an STO:Nb photoanode with a lower $N_{D}$ at $2.0 \times 10^{19} \mathrm{~cm}^{-3}$ (Figure 2b), and further increase to $82 \%(310 \mathrm{~nm})$ and $26 \%(360 \mathrm{~nm})$ for an even lower $N_{D}$ at $1.3 \times 10^{18} \mathrm{~cm}^{-3}$ (Figure 2c). Given the high reflectance of STO in water ( $15 \%$, Supporting Information, Figure S1), these results imply that the carrier separation efficiency is close to unity for the case of lower doping and direct band edge excitation. Within the potential range being studied $(0.48-1.48$ $\mathrm{V}_{\mathrm{RHE}}$ ) the IPCE increases slightly toward higher potential, reflecting the improved minority 
carrier delivery efficiency to the aqueous interface as the depletion region widens, which is consistent to Gärtner's model. ${ }^{[24]}$ It is also notable that $n$-STO photoanodes with similar doping concentration achieve similar IPCE values, regardless of the dopants being niobium atoms (Figure 2c) or oxygen vacancies (Figure 2d), as they both act as shallow donors in STO. ${ }^{[22,23]}$ The only difference is that $n$-STO doped with oxygen vacancies achieves slightly lower IPCE at lower potentials. This may be due to the fact that niobium is a singly charged center but oxygen vacancy is doubly charged, which interact differently with the photogenerated carriers and may have slightly different ionization ratios at room temperature. ${ }^{[23]}$

\subsection{PEC water splitting over STO photoanodes of graded doping}

It is generally accepted that the quantum efficiency of a water splitting photoanode is determined by the depletion width $d_{s c}$ (Figure 3a, inset), as only photoholes generated within the SCR are driven to the semiconductor/water interface without reliance on the slower diffusion process. ${ }^{[24,25]}$ The quantum efficiency is maximized when most incident photons are absorbed within the SCR. Based on the Mott-Schottky relation (Eq. 1) and that the capacitance per unit area of a parallel plate capacitor is $C=\epsilon \epsilon_{0} / d$, we obtain the depletion width

$$
d_{s c}=\epsilon \epsilon_{0} / C_{s c}=\sqrt{2 \epsilon \epsilon_{0}\left(E-E_{F B}-k_{B} T / e\right) / e N_{D}},
$$

which is plotted against $N_{D}$ for $n$-STO in Figure $3 \mathrm{a}$, at $E=1.23 \mathrm{~V}_{\mathrm{RHE}}$. From Figure $3 \mathrm{a}$, it is apparent that lower donor density would be required to achieve high IPCE for lower energy excitation, due to its larger photon attenuation depth. As seen in Figure 3b, although a donor density of $10^{19} \mathrm{~cm}^{-3}$ provides large enough $d_{s c}$ for efficient utilization of $300 \mathrm{~nm}$ excitation, a donor density 10 times lower is necessary for $325 \mathrm{~nm}$, which agrees very well with the actual IPCE measurements shown in Figure 2. It is worth noting that even at a high doping density of $10^{19} \mathrm{~cm}^{-3}$ the depletion region remains as wide as $50 \mathrm{~nm}$; this is due to the high dielectric 
constant of STO $(\epsilon=300)$. As only a tiny fraction $(\sim 0.1 \%)$ of solar photons have energy above STO's direct band gap, for practical reasons we seek to substantially improve the IPCE from indirect band edge excitation. To achieve this requires a donor density of no more than $10^{17} \mathrm{~cm}^{-3}$. However, experimentally it is difficult to produce $n$-STO with $N_{D} \leq 10^{17} \mathrm{~cm}^{-3}$ that remains conductive enough for PEC measurements. The difficulty, as suggested by Frederikse et al, is due to the presence of compensating acceptors or deep trap states that arise from impurities at a comparable concentration. ${ }^{[22]}$

We therefore design an $n$-STO photoanode with its bulk heavily doped for high electron conductivity but its surface lightly doped for larger depletion width. To fabricate such device we start from an $n$-STO photoanode that is uniformly doped by oxygen vacancies $\left(N_{D}=1.7 \times\right.$ $10^{18} \mathrm{~cm}^{-3}$ ) and heat it in oxygen to partially replenish the vacancies and to establish a low doping region near its top surface (Figure 3b). The depth of this low doped region can be controlled by the oxidation temperature and duration, while the back side of the photoanode is protected from oxidation by the aluminum contact. As shown in Figure 3c, the oxidation leads to lower capacitance, implying widened depletion regions and reduced donor densities. The nonlinearity of the Mott-Schottky $1 / C_{s c}^{2}-E$ plots, on the other hand, reflects the nonuniform donor density profile across the electrode's depth. The donor density at a distance $x$ from the interface, $N_{D}(x)$ can be evaluated when the depletion width $d_{\text {sc }}$ equals $x$, using the differential form of Mott-Schottky equation

$$
\left.\frac{\partial\left(1 / C_{S c}^{2}\right)}{\partial E}\right|_{d_{S C}=x}=\frac{2}{e \epsilon \epsilon_{0} N_{D}(x)}
$$

and $C_{S C}=\epsilon \epsilon_{0} / x,{ }^{[26]}$ from which we can determine the $N_{D}(x)-x$ relation for each annealed electrode (Figure 3d). We find that a 1-minute oxidation at $150^{\circ} \mathrm{C}$ is sufficient to create a low doping region $\left(N_{D} \sim 10^{17} \mathrm{~cm}^{-3}\right)$ of $200 \mathrm{~nm}$ thickness near the electrode's top surface and that the donor density gradually approaches the bulk value at larger depth. At higher temperature $\left(200^{\circ} \mathrm{C}\right)$ the lower doping region is widened to about $450 \mathrm{~nm}$ or $700 \mathrm{~nm}$, respectively after a 
1- or 2-minute oxidation. On the other hand, the bulk of the photoanode remains its dark blue appearance and high conductivity of $\sim 2.2 \Omega^{-1} \mathrm{~cm}^{-1}$ (Supporting Information), suggesting that the bulk oxygen vacancies are not affected. It is notable that the oxygen vacancies can be refilled at such low temperature, reflecting its readiness for reoxidation that is consistent with a previous report. ${ }^{[27]}$ The low doping region is considerably more resistive, with an electrical conductivity $\sim 10^{4}$ times lower than the bulk. This occurs despite the apparent donor density being only about 20 times lower (Supporting Information). This strongly implies that the remaining donors at $\sim 10^{17} \mathrm{~cm}^{-3}$ are being compensated by acceptors arising from metal impurities. ${ }^{[22]}$ Elemental analysis supplied by the vendor confirms the presence of metal impurities including iron (Fe) and aluminum ( $\mathrm{Al}$ ) at $1 \mathrm{ppm}$ level, corresponding to a density around $10^{17} \mathrm{~cm}^{-3}$.

As expected, the IPCE due to indirect band gap excitation is significantly improved with the introduction of a low doping region. Prior to oxidation, the uniformly doped $n$-STO $\left(N_{D}=\right.$ $\left.1.7 \times 10^{18} \mathrm{~cm}^{-3}\right)$ exhibits an IPCE of $57 \%(340 \mathrm{~nm})$ and $24 \%(360 \mathrm{~nm})$ at $1.23 \mathrm{~V}_{\mathrm{RHE}}$ (Figure 4a). After oxidation at $150^{\circ} \mathrm{C}$ for 1 minute, which creates a low doping region of $200 \mathrm{~nm}$ wide, the measured IPCE increase to 61\% (340 nm) and 29\% (360 nm) (Figure 4b). Further improvement is made when the low doping region is widened to $\sim 450 \mathrm{~nm}$ by oxidizing the $n$ STO at $200^{\circ} \mathrm{C}$ for 1 minute, with IPCE increased to $74 \%(340 \mathrm{~nm})$ and $46 \%$ (360 nm) (Figure 4c). In this case, the IPCE for excitation near the indirect band edge is doubled from the uniformly doped $n$-STO, and is over 10 times larger than the STO:Nb sample with $N_{D}=3.3 \times$ $10^{19} \mathrm{~cm}^{-3}$. This is consistent with the widening of depletion region. The improvement to IPCE leads to higher photocurrent under simulated AM1.5 solar radiation. Comparing to the uniformly doped $n$-STO, the photocurrent at $1.23 \mathrm{~V}_{\mathrm{RHE}}$ increases by $11 \%$ and $38 \%$, respectively with a low doping region of $200 \mathrm{~nm}$ and $500 \mathrm{~nm}$ thick (Supporting Information, Figure S5).

\subsection{Quantum efficiency as a function of depletion width}


The presence of the low doping region does not improve the quantum efficiency across the entire spectrum. The IPCE for direct transitions $(\lambda \leq 330 \mathrm{~nm})$ drops immediately with the introduction of a low doping region (Figure $4 \mathrm{~b}$ ). Furthermore, when the low doping region is widened beyond $500 \mathrm{~nm}$, IPCE drops across the entire spectral range (Figure 4d). To better illustrate the trend, we summarize the data presented in Figure 2 and Figure 4 by plotting the IPCE of various $n$-STO photoanodes for each excitation wavelength against their respective depletion widths at $1.23 \mathrm{~V}_{\mathrm{RHE}}$ (Figure 5a). It is clear that the IPCE is maximized at a threshold depletion width that is dependent on the excitation wavelength, which is due to carrier recombination within the depletion region. As shown by Jarrett, the photocurrent decreases when the depletion region becomes so wide that it takes longer than its lifetime $\left(\tau_{R}\right)$ for the minority carrier to transit across the depletion region. ${ }^{[28]}$ The transit time $\tau_{t}$ is given by

$$
\tau_{t}=d_{s c}^{2} / 2 \Delta V \mu_{p}
$$

where $d_{s c}$ is the depletion width, $\Delta V$ the magnitude of band bending, and $\mu_{p}$ the minority carrier (hole) mobility. The threshold depletion width is defined when $\tau_{t}$ equals $\tau_{R}$. Using the literature values of $\tau_{R} \sim 1 \mathrm{~ns}$ and $\mu_{p} \sim 1 \mathrm{~cm}^{2} \mathrm{~V}^{-1} \mathrm{~s}^{-1}$ for $n$-STO, ${ }^{[29,30]}$ the threshold width is estimated to be $\sim 500 \mathrm{~nm}$ at $\Delta V=1.23 \mathrm{~V}$, which agrees very well with the case of indirect transitions (Figure 5a). However, the case of direct transitions $(\lambda \leq 330 \mathrm{~nm})$ appears contradictory to the model, as the corresponding threshold width is far below $500 \mathrm{~nm}$ (Figure 5a). Jarrett's model, on the other hand, predicts a threshold width independent of excitation wavelength. In addition, one also expects that the stronger light absorption of the direct transition should always lead to higher efficiency than the indirect transition, which again contradicts the case of $n$-STO photoanodes with a low doping region (Figure $4 \mathrm{~b}-\mathrm{d}$ ).

The apparent contradictions are likely due to the presence of mid-gap charge traps that are not considered in Jarrett's model. The metal impurities contained in our $n$-STO substrates, including $\mathrm{Al}$ and $\mathrm{Fe}$, contribute to a number of mid-gap states. These impurities are 
consistently present at PPM levels in single crystalline STO samples that we have studied from several batches. Our observations are also consistent with transport measurements performed in the 1960s. ${ }^{[22]}$ These impurities may reflect a technical limitation of the crystal growth technique. Using density functional theory $(\mathrm{DFT})^{[31]}$ calculations based on the generalized gradient approximation and using the VASP code $^{[32]}$ with the projectoraugmented wave approach ${ }^{[33]}$ and the standard defect formalism ${ }^{[34]}$ we determine that $\mathrm{Al}$ and Fe impurities most favorably occupy the Ti site in STO and act as acceptors (Supporting Information, Figure S6). By performing hybrid DFT calculations, ${ }^{[35]}$ which provide an accurate description of the bulk STO band structure and are capable of quantitatively predicting defect transition levels, ${ }^{[36]}$ we determine the position of $\mathrm{Al}_{\mathrm{Ti}}$ and $\mathrm{Fe}_{\mathrm{Ti}}$ levels in the STO band gap (Figure $5 \mathrm{~b}$, left side). We find that the $\mathrm{Al}_{\mathrm{Ti}}$ acceptor introduces two levels which are 0.54 and $0.62 \mathrm{eV}$ above the valence-band maximum (VBM) of STO and the $\mathrm{Fe}_{\mathrm{Ti}}$ acceptor introduces two levels near the midgap of STO, at $1.38 \mathrm{eV}$ and $2.20 \mathrm{eV}$ above the VBM, and a third level only $0.02 \mathrm{eV}$ from the conduction-band minimum of STO. Although the mid-gap states are completely occupied at the flat band condition, they are partially vacated in the depletion region and become available for trapping of photoelectrons and act as recombination centers. In the case of direct transitions, the photon attenuation depth $(\sim 100 \mathrm{~nm})$ may be less than the depletion width, leaving the bottom of depletion region under dark (Figure 5b). As a result, the majority carrier (electron) transport within the "dark depletion region" becomes inferior due to the trapping of photoelectrons. The threshold width for direct transition would be comparable to the photon attenuation depth, which is consistent to the experimental observation (Figure 5a). On the contrary, in the case of weak light absorption, photoelectrons are generated across the entire depletion region, which ensures higher carrier mobility and collection efficiency at the cost of less efficient light absorption (Figure 5c).

The schemes depicted in Figures $5 b$ and $5 c$ are further supported by the potential dependence of the IPCE. We find that, for the STO photoanodes with a low doping region 
thicker than $200 \mathrm{~nm}$, the IPCE slightly decreases for direct transitions as the potential moves above $0.75 \mathrm{~V}_{\text {RHE }}$. It implies that the carrier collection efficiency drops with a higher driving force for carrier separation, which appears abnormal but is indeed consistent with Figure 5b. In the case of strong light absorption and very wide depletion region, further widening of the depletion region no longer increases light absorption, but empties more mid-gap trap states and diminishes the collection efficiency of photoelectrons. The behavior for indirect transition $(\lambda>330 \mathrm{~nm})$, on the other hand, remains "normal" as predicted by Jarrett's model.

\section{Conclusion}

In summary, efficient water splitting at planar semiconductor photoelectrodes has been robustly extended into the wavelength regime where there is only indirect band gap excitation with correspondingly weak optical absorption. By creating a lower doping region of tunable depth over a single crystalline $n$-STO photoanode using a newly developed re-oxidation technique, the depletion region in $n$-STO is widened to beyond $500 \mathrm{~nm}$, a value unprecedented for a metal oxide photoanode. The dramatically improved optical absorption within the widened depletion region leads to very high IPCE $(>70 \%)$ for excitation below the STO direct band edge, which is comparable to the best results obtained for excitation energies above the direct band edge. This strategy offers a new path for optimizing the potential for visible light water splitting photocatalysts such as $\mathrm{WO}_{3}$ and $\mathrm{Fe}_{2} \mathrm{O}_{3}$, which share a similar problem of having an indirect band gap. However, the benefit derived from widening the depletion region can be limited by the presence of impurities that contribute to charge traps in the depletion region, even at a very low concentration $(\sim 1 \mathrm{ppm})$. This hinders the collection of photoelectrons in the quasi-neutral region. This observation strongly suggests that semiconductors of high quality and high purity will be required to release the full potential for photoelectrochemical water splitting in these materials.

\section{Experimental Section}


Preparation of n-STO photoanodes: In all of our studies, single crystalline pristine STO and niobium doped $n$-STO (STO:Nb) substrates are obtained from a commercial source (MTI Corporation, USA) and are grown by the Verneuil process. As received, the substrates are cut along the (100) facets and polished so as to be epitaxy-ready. Pristine STO substrates are $n$ type doped with oxygen vacancies by heating in a high vacuum chamber (PVD Products, USA) at $900^{\circ} \mathrm{C}$ for 10 hours, at a base pressure lower than $10^{-7}$ Torr. In this process, the transparent and insulating substrates become dark blue in color, with a typical conductivity of $(2.2 \pm 0.3) \mathrm{S} \cdot \mathrm{cm}^{-1}$ (Supporting Information). A photoanode is fabricated by depositing a 75 nm-thick aluminum layer to the back side of an $n$-STO substrate, through thermal evaporation (K. J. Lesker PVD75), which establishes a reliable ohmic contact to the substrates. Aluminum is chosen as the contact metal to match the low work function of $n$-STO. ${ }^{[37]}$

STO photoanode of a graded doping profile is prepared by heating an $n$-STO photoanode that is uniformly doped by oxygen vacancies $\left(N_{D}=1.7 \times 10^{18} \mathrm{~cm}^{-3}\right)$ in oxygen to partially replenish the vacancies near its top surface. The process is conducted in a rapid thermal processor (RTP 600S, Modular Pro). Under an oxygen flow of 3 SLPM, the substrate is heated to the processing temperature $\left(150-200^{\circ} \mathrm{C}\right)$ at a ramp rate of $15^{\circ} \mathrm{C} \mathrm{s}^{-1}$ for a specified duration and is quickly cooled afterwards. The depth of the low doped region can be controlled by the oxidation temperature and duration, while the back side of the photoanode is protected from oxidation by the aluminum contact.

Photoelectrochemical characterization: PEC measurements are performed by a potentiostat (VersaStat4, PAR) in standard three-electrode configuration, with a STO photoanode placed in a custom-built quartz-windowed PEC cell as the working electrode. The active/uniform illumination area is $0.18 \mathrm{~cm}^{2}$. The reference electrode is an $\mathrm{Ag} / \mathrm{AgCl} / 3 \mathrm{M} \mathrm{KCl}$ Dri-Ref electrode $\left(0.210 \mathrm{~V}_{\mathrm{NHE}}\right.$, WPI Inc.) and the counter electrode is a platinum wire. The electrolyte is an aqueous solution of $0.1 \mathrm{~m} \mathrm{KOH}$. Monochromatic illumination for the IPCE measurements is provided by a $300 \mathrm{~W}$ xenon arc lamp (Newport) and a 1/8 $\mathrm{m}$ grating 
monochromator (Newport CS130) equipped with band pass filters for removing higher order diffractions. The light power for each wavelength is measured by an optical power meter (Newport 1918-C) and a UV-enhanced Si photodiode sensor.

\section{Supporting Information}

Supporting Information is available from the Wiley Online Library or from the author.

\section{Acknowledgements}

This research used resources of the Center for Functional Nanomaterials, which is a U.S.

DOE Office of Science User Facility, at Brookhaven National Laboratory under Contract No.

DE-SC0012704. This research used resources of the National Energy Research Scientific

Computing Center, a DOE Office of Science User Facility supported by the Office of Science of the U.S. Department of Energy under Contract No. DE-AC02-05CH11231. We thank Dr. Charles T. Black for helpful discussion.

Received: ((will be filled in by the editorial staff))

Revised: ((will be filled in by the editorial staff)) Published online: ((will be filled in by the editorial staff))

[1] A. Fujishima, K. Honda, Nature 1972, 238, 37.

[2] M. G. Walter, E. L. Warren, J. R. McKone, S. W. Boettcher, Q. X. Mi, E. A. Santori, N. S. Lewis, Chem. Rev. 2010, 110, 6446.

[3] A. J. Nozik, Ann. Rev. Phys. Chem. 1978, 29, 189.

[4] K. Appavoo, M. Liu, C. T. Black, M. Y. Sfeir, Nano Lett. 2015, 15, 1076.

[5] M. N. Huda, A. Walsh, Y. F. Yan, S. H. Wei, M. M. Al-Jassim, J. Appl. Phys. 2010, 107, 123712.

[6] D. C. Cronemeyer, Phys. Rev. 1952, 87, 876.

[7] A. J. E. Rettie, H. C. Lee, L. G. Marshall, J. F. Lin, C. Capan, J. Lindemuth, J. S. McCloy, J. S. Zhou, A. J. Bard, C. B. Mullins, J. Am. Chem. Soc. 2013, 135, 11389.

[8] M. Z. Liu, N. D. Snapp, H. Park, Chem. Sci. 2011, 2, 80.

[9] B. Liu, C. H. Wu, J. W. Miao, P. D. Yang, ACS Nano 2014, 8, 11739.

[10] F. F. Abdi, L. H. Han, A. H. M. Smets, M. Zeman, B. Dam, R. van de Krol, Nat. Commun. 2013, 4, 2195. 
[11] L. H. Han, F. F. Abdi, R. van de Krol, R. Liu, Z. Q. Huang, H. J. Lewerenz, B. Dam, M. Zeman, A. H. M. Smets, Chemsuschem 2014, 7, 2832.

[12] I. Cesar, K. Sivula, A. Kay, R. Zboril, M. Graetzel, J. Phys. Chem. C 2009, 113, 772.

[13] L. A. Marusak, R. Messier, W. B. White, J. Phys. Chem. Solids 1980, 41, 981.

[14] F. P. Koffyberg, K. Dwight, A. Wold, Solid State Commun. 1979, 30, 433.

[15] S. K. Deb, Philos. Mag. 1973, 27, 801.

[16] K. van Benthem, C. Elsasser, R. H. French, J. Appl. Phys. 2001, 90, 6156.

[17] M. I. Cohen, R. F. Blunt, Phys. Rev. 1968, 168, 929.

[18] G. E. Jellison, I. Paulauskas, L. A. Boatner, D. J. Singh, Physical Review B 2006, 74, 155130.

[19] M. S. Wrighton, A. B. Ellis, P. T. Wolczanski, D. L. Morse, H. B. Abrahamson, D. S. Ginley, J. Am. Chem. Soc. 1976, 98, 2774.

[20] F. T. Wagner, G. A. Somorjai, Nature 1980, 285, 559.

[21] T. K. Townsend, N. D. Browning, F. E. Osterloh, ACS Nano 2012, 6, 7420.

[22] H. P. R. Frederikse, W. R. Thurber, W. R. Hosler, Phys. Rev. 1964, 134, A442.

[23] O. N. Tufte, P. W. Chapman, Phys. Rev. 1967, 155, 796.

[24] W. W. Gärtner, Phys. Rev. 1959, 116, 84.

[25] R. v. d. Krol, M. Grätzel, Photoelectrochemical hydrogen production, Springer, New York 2012.

[26] A. T. R. Briggs, J. P. Stagg, Semicond. Sci. Tech. 1988, 3, 469.

[27] F. D. Gealy, H. L. Tuller, J. Phys. Colloques 1990, 51, C1.

[28] H. S. Jarrett, J. Appl. Phys. 1981, 52, 4681.

[29] Q.-L. Zhou, K.-J. Jin, H.-B. Lu, P. Han, Z.-H. Chen, K. Zhao, Y.-L. Zhou, G.-Z. Yang, EPL (Europhys. Lett.) 2005, 71, 283.

[30] M. Fleischer, H. Meixner, C. Tragut, J. Am. Ceram. Soc. 1992, 75, 1666.

[31] W. Kohn, L. J. Sham, Phys. Rev. 1965, 140, A1133.

[32] G. Kresse, D. Joubert, Phys. Rev. B 1999, 59, 1758.

[33] P. E. Blöchl, Phys. Rev. B 1994, 50, 17953.

[34] C. Freysoldt, B. Grabowski, T. Hickel, J. Neugebauer, G. Kresse, A. Janotti, C. G.

Van de Walle, Rev. Mod. Phys. 2014, 86, 253.

[35] J. Heyd, G. E. Scuseria, M. Ernzerhof, J. Chem. Phys. 2003, 118, 8207.

[36] A. Janotti, J. B. Varley, M. Choi, C. G. van de Walle, Phys. Rev. B 2014, 90, 085202.

[37] T. Shimizu, N. Gotoh, N. Shinozaki, H. Okushi, Appl. Sur. Sci. 1997, 117, 400. 

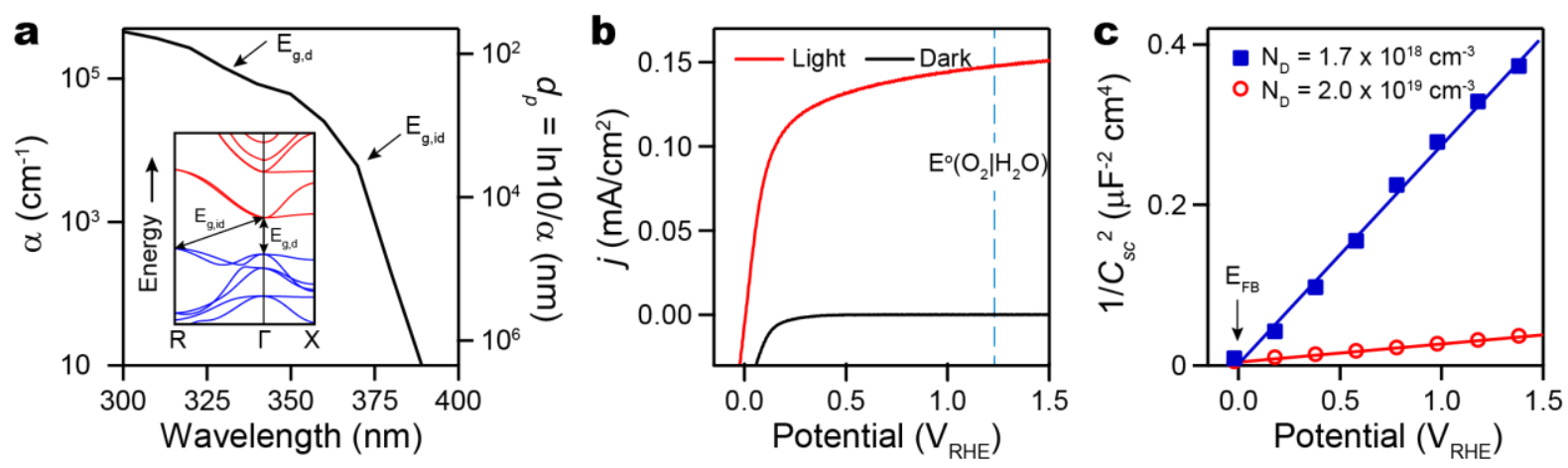

Figure 1. a, The wavelength dependent STO optical absorption coefficient (left axis) and corresponding photon attenuation length (right axis). The inset is the DFT band structure (partial) of STO that shows the direct $\left(E_{g, d}\right)$ and indirect transitions $\left(E_{g, i d}\right)$. b, Current density $v s$ potential curves for an $n$-STO photoanode in dark (black) and under white light excitation (red). The thermodynamic potential for oxygen evolution $\left(E^{\circ}\left(\mathrm{O}_{2} \mid \mathrm{H}_{2} \mathrm{O}\right)\right)$ is indicated by a dashed vertical line. c, Mott-Schottky plots for two $n$-STO photoanodes with different donor densities. Solid colored lines correspond to linear fits to the data. 


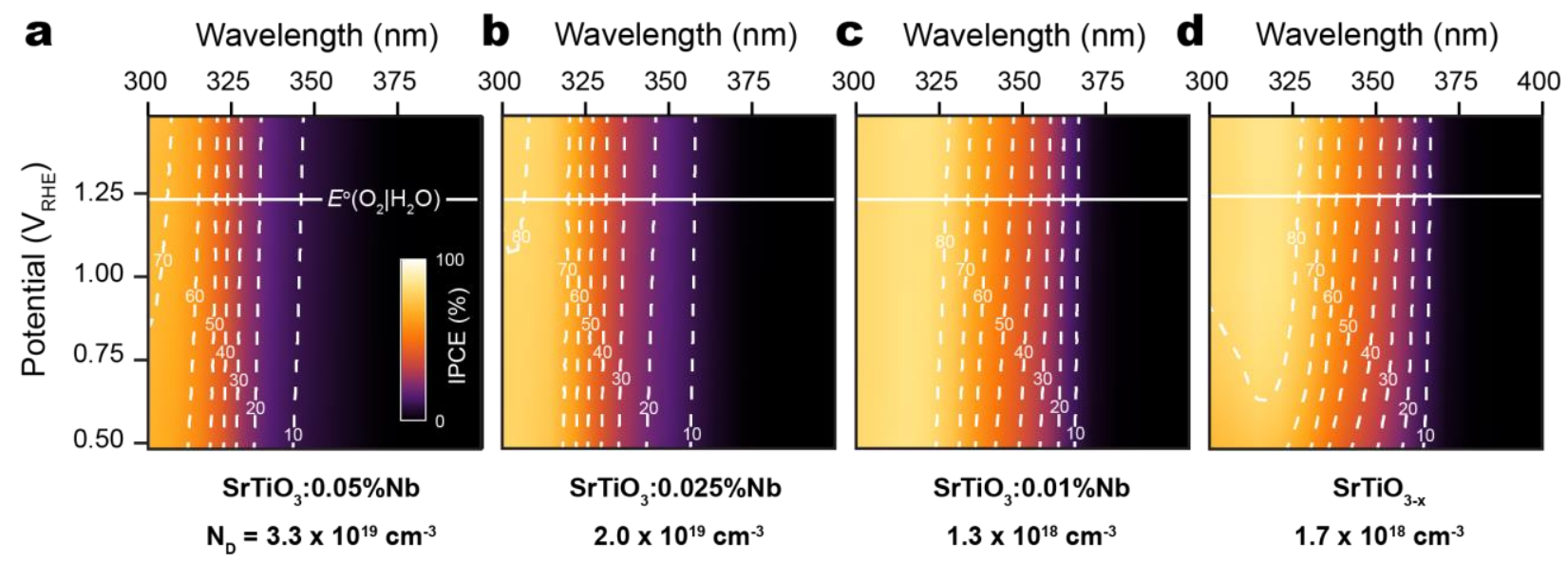

Figure 2. a-d, IPCE as a function of excitation wavelength and electrode potential, for four $n$ STO photoanodes with various dopant types (niobium or oxygen vacancies) and donor densities. The dashed lines are contours for corresponding IPCE values in percentage (\%). All the panels share the same color scale. The horizontal white lines indicate the thermodynamic potential for oxygen evolution $\left(E^{\circ}\left(\mathrm{O}_{2} \mid \mathrm{H}_{2} \mathrm{O}\right)\right)$ at $1.23 \mathrm{~V}_{\text {RHE }}$. 

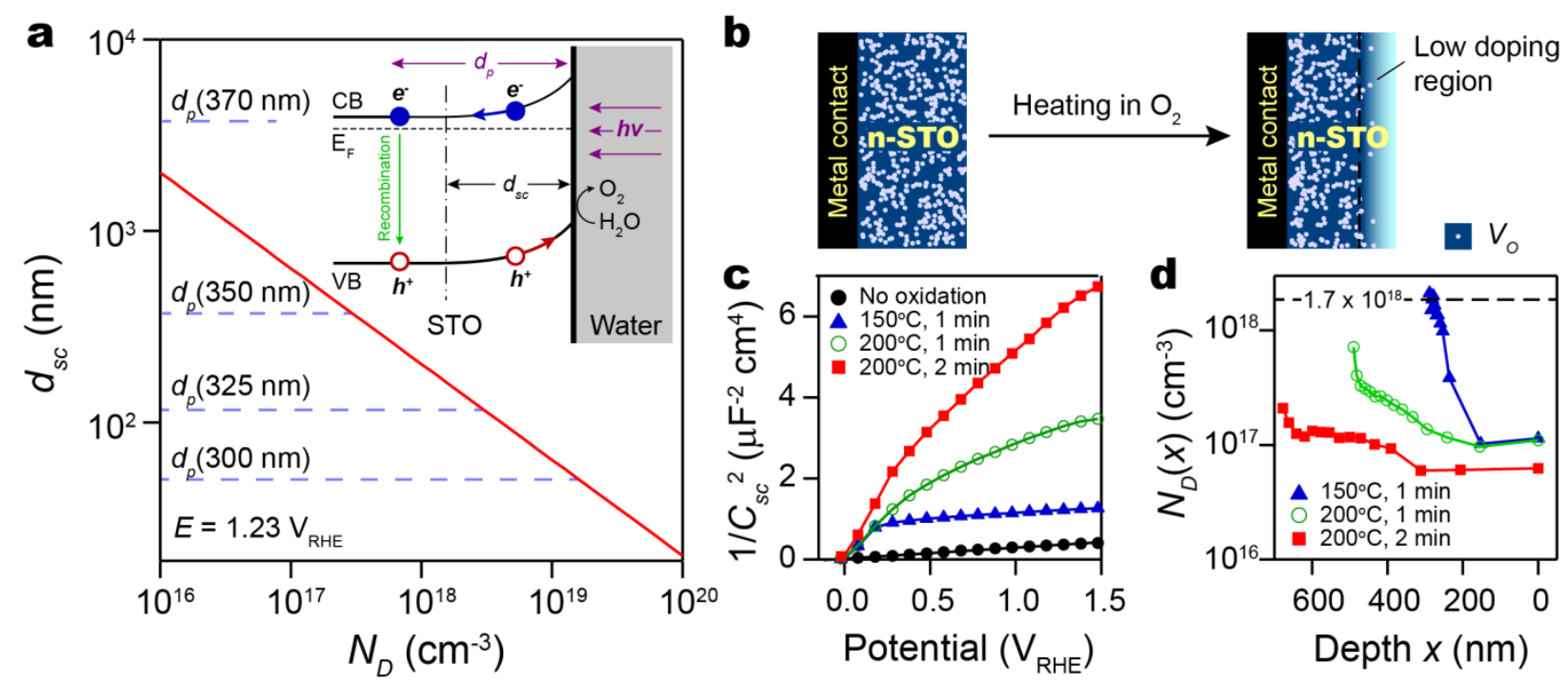

Figure 3. a, The depletion width $d_{s c}$ as a function of donor density for the $n$-STO|water interface, with band bending $\Delta \phi=1.23 \mathrm{~V}$. The horizontal dashed lines indicate the photon attenuation depth $\left(d_{p}\right)$ in STO for a few representative wavelengths. (inset) A simplified illustration for photocarrier dynamics near the $n$-STO|water interface. $\mathbf{b}$, A low doping region is created over the $n$-STO photoanode by refilling the oxygen vacancies $\left(V_{o}\right)$ through thermal oxidation. c, Mott-Schottky plots for a uniformly-doped $n$-STO photoanode (black dots) and the ones re-oxidized under various conditions. d, Donor density versus depth, i.e., distance from the top surface, for $n$-STO photoanode re-oxidized under various conditions. 


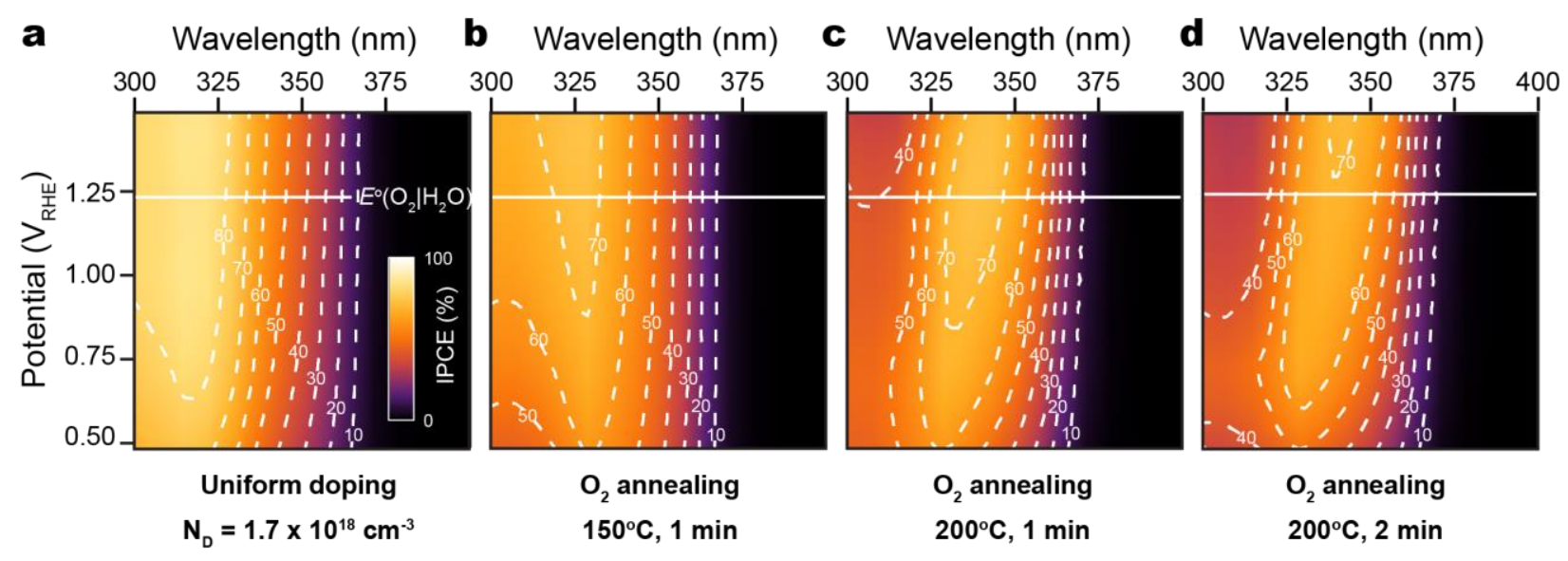

Figure 4. IPCE as a function of excitation wavelength and electrode potential, for a uniformly-doped $n$-STO photoanode (a) and the ones re-oxidized under various conditions (bd). The dashed lines are contours for corresponding IPCE values in percentage (\%). All the panels share the same color scale. The horizontal white lines indicate the thermodynamic potential for oxygen evolution $\left(E^{\circ}\left(\mathrm{O}_{2} \mid \mathrm{H}_{2} \mathrm{O}\right)\right)$ at $1.23 \mathrm{~V}_{\mathrm{RHE}}$. 

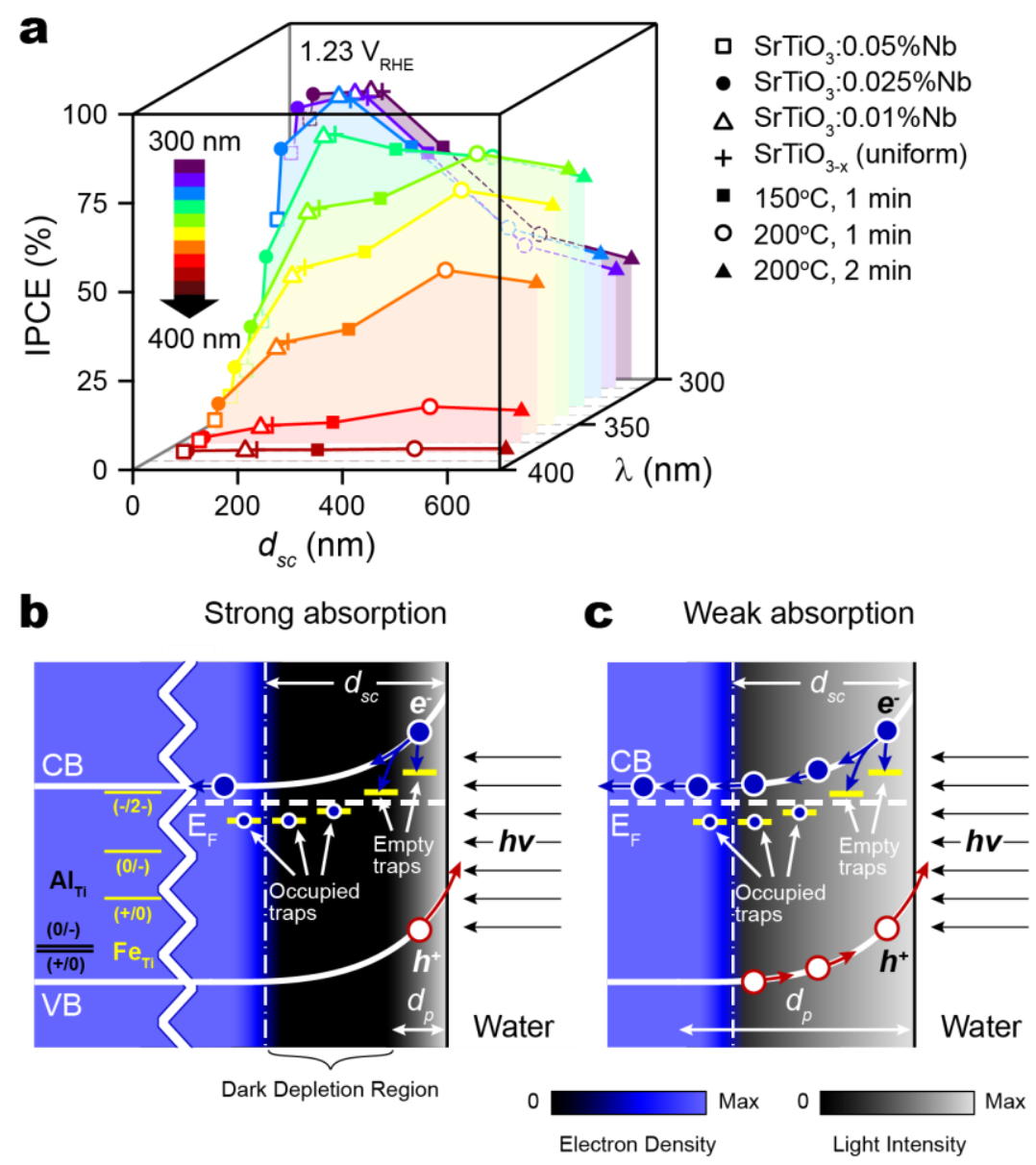

Figure 5. a, IPCE values of various $n$-STO photoanodes at $1.23 \mathrm{~V}_{\mathrm{RHE}}$ versus their respective depletion widths $\left(d_{s c}\right)$ for several excitation wavelengths between $300 \mathrm{~nm}$ and $400 \mathrm{~nm}$. The data collected from the same sample are labeled by symbols of the same shape. $\mathbf{b}$, In the case of strong light absorption $\left(d_{s c} \gg d_{p}\right)$, carriers are generated only near the top of the depletion region. The presence of mid-gap acceptors can easily trap the photoelectrons, leading to lower carrier collection efficiency. The calculated acceptor levels of $\mathrm{Al}_{\mathrm{Ti}}$ and $\mathrm{Fe}_{\mathrm{Ti}}$ in $\mathrm{STO}$ are shown in the left side. c, In the case of weak light absorption $\left(d_{s c} \sim d_{p}\right)$, carrier are generated across the entire depletion. The collection of photoelectrons is therefore affected much less by the trap states. 


\section{TABLE OF CONTENTS ENTRY}

Water photolysis over semiconducting metal oxide is studied at the limit of very wide depletion region, for $n-\mathrm{SrTiO}_{3}$ photoanodes. Through a graded doping architecture, the depletion region is widened to an unprecedented depth of $500+\mathrm{nm}$, which optimizes the trade-off between photon collection and minority carrier delivery, leading to an IPCE over $70 \%$ for its weak indirect band gap absorption.

Keywords: photoelectrochemical water splitting; indirect band gap semiconductor; strontium titanate; graded doping

Mingzhao Liu*, John L. Lyons, Danhua Yan, and Mark S. Hybertsen

Semiconductor-based Photoelectrochemical Water Splitting at the Limit of Very Wide Depletion Region
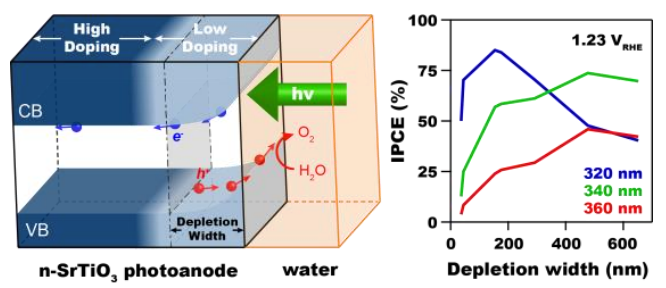


\section{Supporting Information}

\section{Semiconductor-based Photoelectrochemical Water Splitting at the Limit of Very Wide Depletion Region}

Mingzhao Liu*, John L. Lyons, Danhua Yan, and Mark S. Hybertsen

Center for Functional Nanomaterials, Brookhaven National Laboratory, Upton, NY 11973, United States

\section{Reflectivity of STO}

At normal incidence, the reflectivity $R$ of an $n$-STO photoanode immersed in water is calculated using the Fresnel equation,

$$
R=\left|\frac{n_{\mathrm{STO}}-n_{\mathrm{H}_{2} \mathrm{O}}}{n_{\mathrm{STO}}+n_{\mathrm{H}_{2} \mathrm{O}}}\right|^{2} .
$$

The complex index of refraction of STO, $n_{\mathrm{STO}}$, is obtained by ellipsometry measurements, using a J. A. Woollam M-2000 spectroscopic ellipsometer (Figure S1a). For the spectral range of interest $(300-400 \mathrm{~nm})$, dispersion can be neglected for water and we use $n_{\mathrm{H}_{2} \mathrm{O}}=1.33$. The reflectivity $\mathrm{R}$ is plotted against wavelength in Figure S1b. It can be seen that the reflectivity of STO in water is close to $20 \%$ above its direct band gap, and gradually drops for longer wavelength illumination. 

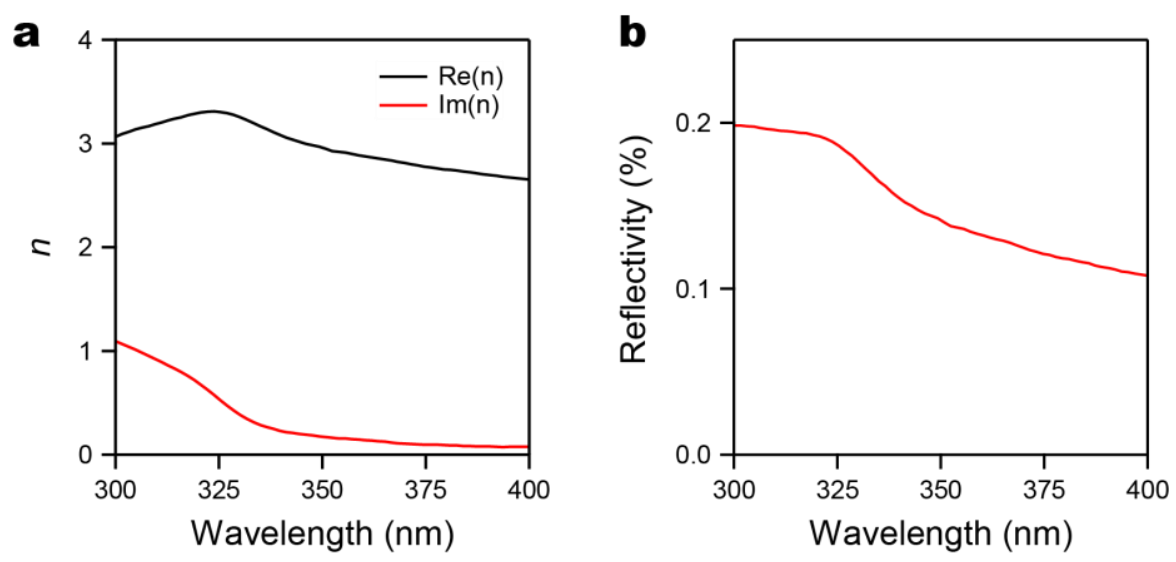

Figure S1. (a) The complex index of refraction of STO, as a function of wavelength. (b) The optical reflectivity of STO immersed in water for normal incidence, as a function of wavelength. 


\section{Mott-Schottky analysis}

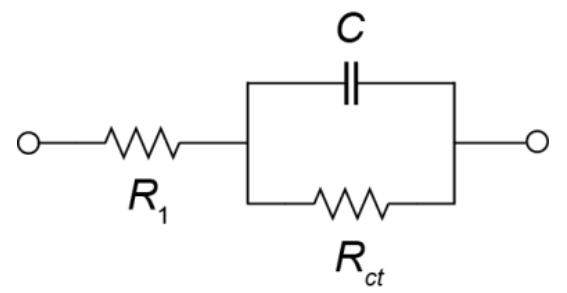

Figure S2. The equivalent circuit used to model the electrochemical impedance of the cell.

The impedance of the electrochemical cell $(Z)$ is measured under dark conditions at a series of electrode potentials, in the frequency $(f)$ range $10^{2}$ to $10^{4} \mathrm{~Hz}$. To extract the space-charge region capacitance from the experimental impedance, the electrochemical cell is modeled by the equivalent circuit shown in Figure S2. ${ }^{[1]}$ The current passing through the cell contributes in parallel to the faradic process that is represented by the charge transfer resistance $R_{c t}$ and nonfaradic interface charging that is represented by $C$, the capacitance per unit area of the aqueous interface. The two current components flow together through the electrolyte solution and the electrical contact to the working electrode, which together are represented by a series resistance $R_{1}$. The capacitance $C$ includes the space-charge region capacitance $\left(C_{s c}\right)$ and the double-layer capacitance $\left(C_{d l}\right)$ in series, $1 / C=1 / C_{s c}+1 / C_{d l}$. Since $C_{d l}$ is usually orders of magnitude larger than $C_{s c}$, the total capacitance $C$ roughly equals to $C_{s c}{ }^{[2]}$ The total impedance of the equivalent circuit is

$$
Z(\omega)=R_{1}+\frac{R_{c t} Z_{C}}{R_{c t}+Z_{C}}=R_{1}-\frac{i R_{c t} / \omega C_{s c} A}{R_{c t}-i / \omega C_{s c} A}=R_{1}-\frac{i R_{c t} / \omega C_{s c} A}{R_{c t}-i / \omega C_{s c} A}
$$

where $\omega=2 \pi f$ is the angular frequency, $A$ is the area of the interface, and $Z_{C}=-i / \omega C_{s c} A$ is the capacitor impedance. The real and imaginary parts of the complex impedance are thus,

$$
\operatorname{Re}(Z)=R_{1}+\frac{R_{c t}}{1+\omega^{2} R_{c t}^{2} C_{s c}^{2} A^{2}}
$$




$$
\operatorname{Im}(Z)=-\frac{\omega R_{c t}^{2} C_{s c} A}{1+\omega^{2} R_{c t}^{2} C_{s c}^{2} A^{2}}
$$

We have a surface area $A=0.18 \mathrm{~cm}^{2}$ for the $\mathrm{STO}$ electrode. The experimental data shown in Figure S2 is then fit to Eqs. S2 and S3 using the EIS Spectrum Analyser, software for analysis of impedance spectrum. ${ }^{[3]}$ The capacitances at other potentials are obtained following the same procedure and are presented in Figure 1(c) and Figure 3(c). 


\section{Electrical conductivity of STO substrates}

To measure the electrical conductivity of freshly reduced $n$-STO substrates, an array of aluminum contact pads is defined by vacuum thermal evaporation with a shadow mask. Each contact pad is $1.3 \mathrm{~mm}$ in diameter and $75 \mathrm{~nm}$ in thickness, with center-to-center distance 1.875 $\mathrm{mm}$ (Figure S3). Two-probe measurements give the resistance between two nearest contacts is $R$ $=4.0 \pm 0.5 \Omega$. Four-probe measurements return a similar value, indicating that the contact resistance is negligible. As the conductive channel does not have a simple shape, the substrate conductivity is calculated using a COMSOL Multiphysics simulation, based on the actual geometry of the device. It is found that with the substrate conductivity of $2.2 \pm 0.3 \mathrm{~S} \cdot \mathrm{cm}^{-1}$, a value that is within the expected range, the experimentally measured resistance is reproduced. Given the substrate donor density of $1.7 \times 10^{18} \mathrm{~cm}^{-3}$ we then obtain the electron mobility of $(8.0$ $\pm 1.1) \mathrm{cm}^{2} \mathrm{~V}^{-1} \mathrm{~s}^{-1}$ for $n$-STO, which is very close to its literature value.

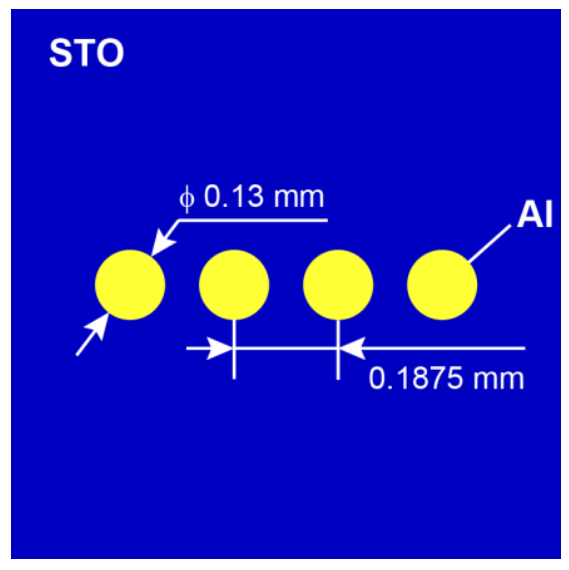

Figure S3. Device geometry for measuring the electrical conductivity of an $n$-STO substrate. The aluminum (Al) contacts are defined by thermal evaporation of aluminum, using a shadow mask. 
To study the bulk and surface conductivity of the $n$-STO substrate bearing a surface low doping region, we start from a freshly reduced $n$-STO substrate and deposit aluminum contacts in a same way as previous study (Figure S4a, contacts 1 and 2). The substrate is then heated in oxygen to generate a low doping region at its surface (Figure S4b). The area under the aluminum contacts, however, is protected from oxidation, as the electrical resistance between contacts 1 and 2 does not change before and after heating in oxygen and remains about $4 \Omega$. It is therefore confirmed that the low-temperature oxidation process does not change the bulk conductivity or carrier density of the n-STO substrate. After this, another set of aluminum contacts (Figure S4c, contacts 3 and 4) are deposited over the low doping region. Resistance measurements indicate that the $700 \mathrm{~nm}$-thick low doping region gives an additional resistance of about $31 \Omega$ at each contact. We can therefore calculate the low doping region's conductivity to be $1.7 \times 10^{-4} \mathrm{~S} \cdot \mathrm{cm}^{-1}$, which is 4 orders of magnitude less than the bulk.

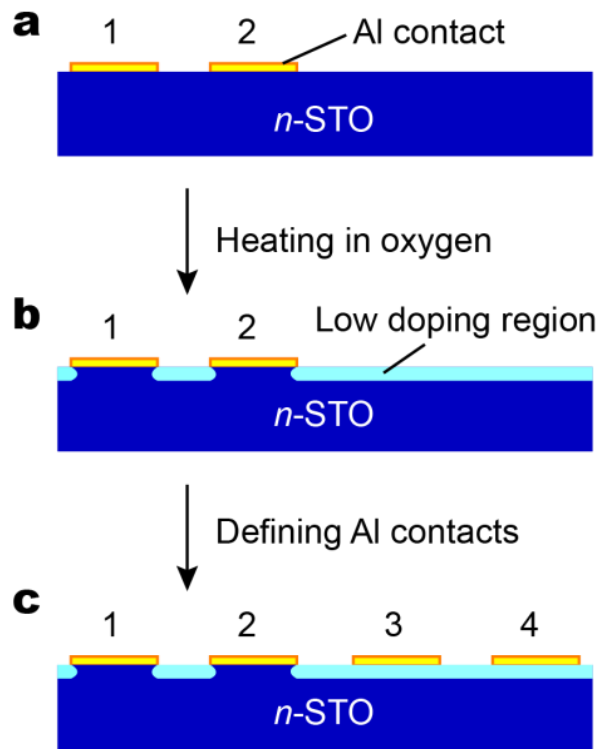

Figure S4. Device fabrication flow for measuring the bulk and surface conductivity of the $n$ STO substrate with a surface low doping region. 


\section{PEC measurement under simulated solar radiation}

The measurements are performed for $n$-STO photoanodes with either uniform doping $\left(N_{D}=1.7\right.$ $\times 10^{18} \mathrm{~cm}^{-3}$ ) or a low doping region of various depths near the aqueous interface. The measurement station is equipped with a potentiostat (PAR VersaStat), a custom-built threeelectrode quartz-windowed PEC cell, and a $150 \mathrm{~W}$ solar simulator with AM 1.5G filter (Newport). In the PEC cell, we use a $0.1 \mathrm{M} \mathrm{KOH}$ solution as an electrolyte, the $n$-STO substrate as a working electrode (active/uniform illumination area of $0.18 \mathrm{~cm}^{2}$ ), an $\mathrm{Ag} / \mathrm{AgCl} / 3 \mathrm{M} \mathrm{KCl}$ reference electrode $\left(0.210 \mathrm{~V}_{\mathrm{NHE}}\right)$, and a platinum wire counter electrode. The incident light power is calibrated by a calibrated quartz-windowed Si solar cell (Newport) and a spectrometer calibrated for an absolute irradiance measurement (Ocean Optics). The $j-E$ relations for the various samples are shown in Figure S5.

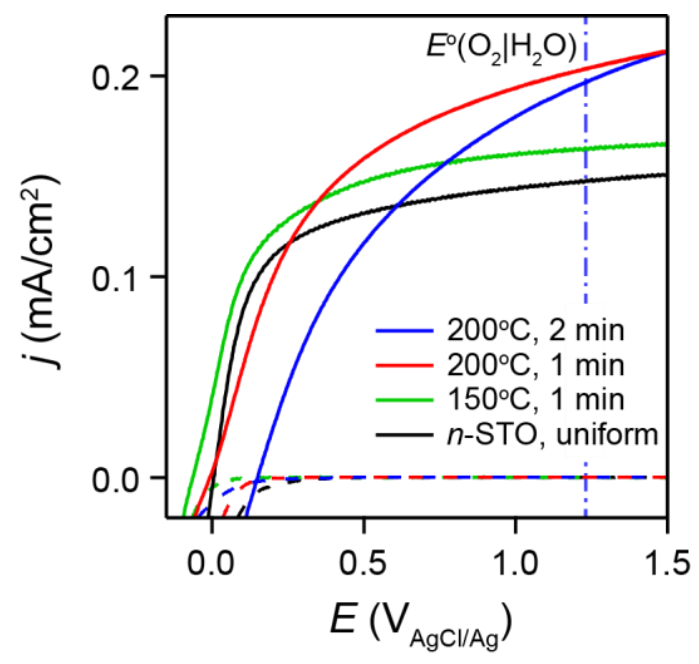

Figure S5. Current density versus potential curves in the dark (dashed lines) and in simulated AM 1.5 illumination (solid lines) for $n$-STO photoanodes with either uniform doping $\left(N_{D}=1.7 \times\right.$ $10^{18} \mathrm{~cm}^{-3}$ ) or a low doping region of various depth. 


\section{DFT calculations}

In order to investigate how $\mathrm{Al}$ and Fe impurities incorporate into the STO crystal structure, we perform calculations based on density functional theory (DFT), ${ }^{[4]}$ with the VASP $\operatorname{code}{ }^{[5]}$ and the projector-augmented wave approach ${ }^{[6]}$. We employ 135-atom cubic supercells and use the standard defect formalism, ${ }^{[7]}$ using a $2 \times 2 \times 2$ special $k$-point mesh and a $400 \mathrm{eV}$ cutoff. Spin polarization is included when unpaired spins are present, and charge-state corrections are included by comparing the averaged electrostatic potential far from the defect site with that of the bulk. The stability of impurity species depend on the chemical potentials of species involved; here we take $\mu_{\mathrm{O}}=-3.1 \mathrm{eV}, \mu_{\mathrm{Sr}}=-4.0 \mathrm{eV}$, and $\mu_{\mathrm{Ti}}=-3.4 \mathrm{eV} \cdot{ }^{[8]}$ The chemical potentials of the impurities are determined by referring to their native oxides, $\mathrm{Al}_{2} \mathrm{O}_{3}$ for $\mathrm{Al}$ and $\mathrm{Fe}_{2} \mathrm{O}_{3}$ for $\mathrm{Fe}$.

Our results are shown in Figure S6. For both impurities we find that occupation of the Ti is far more favorable than occupation of the Sr site. Under $n$-type conditions (when the Fermi level is near the $\mathrm{CBM}$ at $1.73 \mathrm{eV}$ ), $\mathrm{Fe}$ is $2.5 \mathrm{eV}$ more stable occupying the $\mathrm{Ti}$ site over the $\mathrm{Sr}$ site, while $\mathrm{Al}$ is over $6 \mathrm{eV}$ more stable occupying the Ti site over the $\mathrm{Sr}$ site. $\mathrm{Fe}_{\mathrm{Ti}}$ introduces three levels into the $\mathrm{STO}$ band gap while $\mathrm{Al}_{\mathrm{Ti}}$ is a single acceptor. Due to atomic size considerations, neither impurity is expected to occupy the interstitial or O lattice sites.

In order to correct the bulk band structure and accurately predict acceptor transition levels, we then repeat the calculations of $\mathrm{Fe}_{\mathrm{Ti}}$ and $\mathrm{Al}_{\mathrm{Ti}}$ using the screened hybrid functional of Heyd, Scuseria, and Ernzerhof. ${ }^{[9]}$ As with the standard DFT calculations, we find that $\mathrm{Fe}_{\mathrm{Ti}}$ gives rise to three deep transition levels, two that lie near midgap and a third near the CBM. These levels are derived from tightly-bound, Fe-derived $3 d$ orbitals. The charge states of the Fe impurity calculated here are similar to earlier tight-binding results. ${ }^{[10]}$ Applying the hybrid functional we now find that $\mathrm{Al}_{\mathrm{Ti}}$ gives rise to acceptor transition levels near the VBM, whereas from DFT it 
was predicted to be a shallow acceptor. As these levels are derived from O $2 p$ states, the HSEcalculated $\mathrm{Al}_{\mathrm{Ti}}$ transition levels are due to hole trapping at $\mathrm{O}$ sites nearest to the $\mathrm{Al}$ acceptor, an effect that cannot be captured with standard DFT. ${ }^{[11]}$
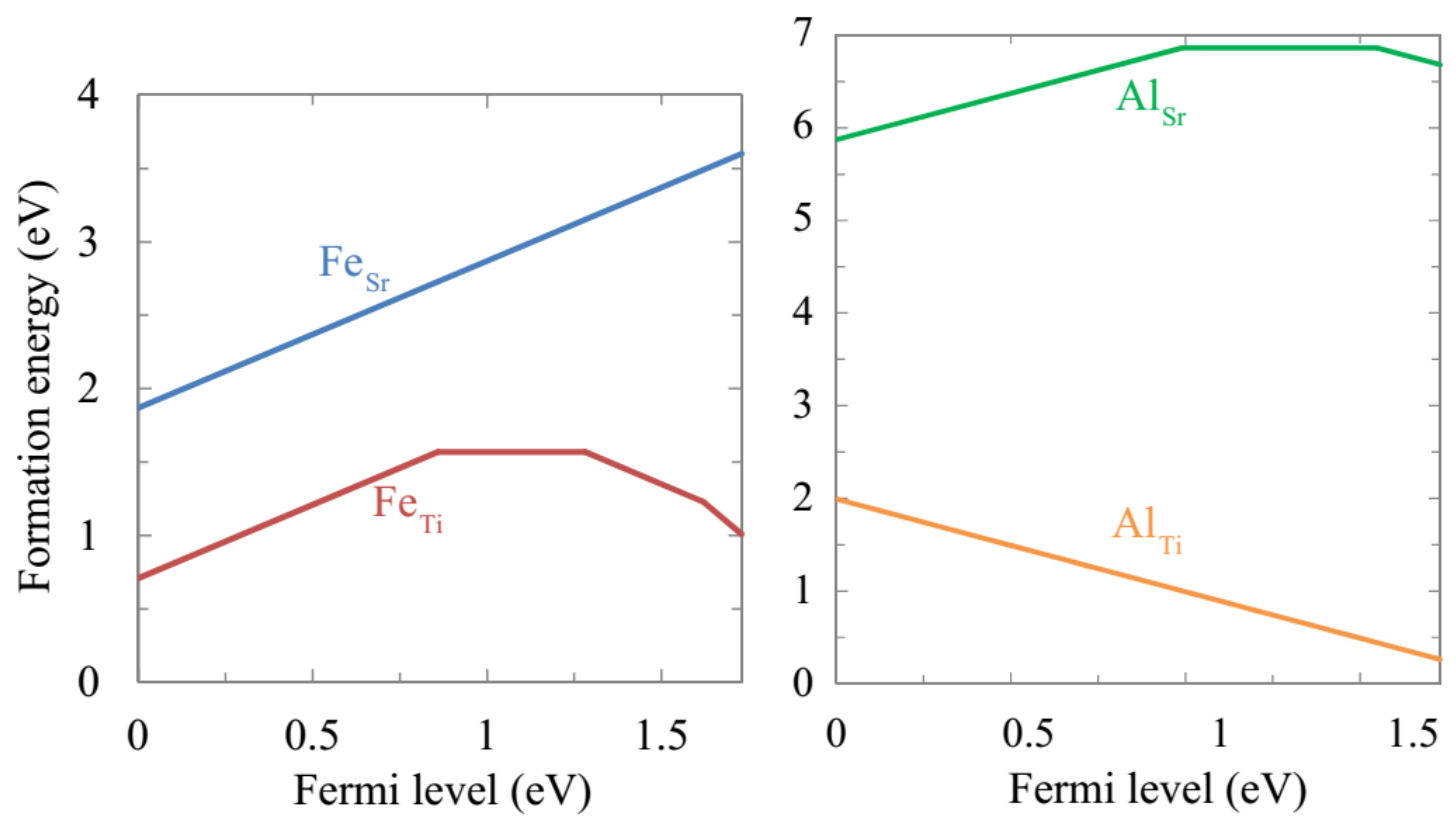

Figure S6. Formation energy versus Fermi level for Al and Fe impurities in STO as calculated within GGA-DFT under the chemical potential conditions described in the text. For both impurities, occupation of the Ti site is favored over the $\mathrm{Sr}$ site. 


\section{References}

[1] A. J. Bard, L. R. Faulkner, Electrochemical methods: fundamentals and applications, Wiley, New York 1980.

[2] M. Grätzel, Nature 2001, 414, 338.

[3] A. S. Bondarenko, G. A. Ragoisha, in Progress in Chemometrics Research, (Ed: A. L. Pomerantsev), Nova Science Publishers, New York 2005, 89.

[4] W. Kohn, L. J. Sham, Phys. Rev. 1965, 140, A1133.

[5] G. Kresse, D. Joubert, Phys. Rev. B 1999, 59, 1758.

[6] P. E. Blöchl, Phys. Rev. B 1994, 50, 17953.

[7] C. Freysoldt, B. Grabowski, T. Hickel, J. Neugebauer, G. Kresse, A. Janotti, C. G. Van de Walle, Rev. Mod. Phys. 2014, 86, 253.

[8] A. Janotti, J. B. Varley, M. Choi, C. G. van de Walle, Phys. Rev. B 2014, 90, 085202.

[9] J. Heyd, G. E. Scuseria, M. Ernzerhof, J. Chem. Phys. 2003, 118, 8207.

[10] M. O. Selme, P. Pecheur, G. Toussaint, J. Phys. C Solid State Phys. 1984, 17, 5185.

[11] J. L. Lyons, A. Janotti, C. G. Van de Walle, J. Appl. Phys. 2014, 115, 012014. 